\title{
Lung neutrophils on a paleo diet: lean, mean inflammatory machines
}

\author{
Ian M. Cartwright and Sean P. Colgan
}

Department of Medicine and the Mucosal Inflammation Program, University of Colorado School of Medicine, Anschutz Medical Campus, Aurora, Colorado, USA.

\begin{abstract}
Sites of acute inflammation become austere environments for the procurement of energy. The combination of oxygen depletion (hypoxia) and decreased glucose availability requires surprising metabolic adaptability. In this issue of the $J C l$, Watts et al. examined the metabolic adaptability of murine neutrophils to the setting of acute pulmonary inflammation elicited by exposure to nebulized endotoxin. While neutrophils are generally considered a primarily glycolytic cell type, Watts et al. used a combination of labeled amino acids and high-resolution proteomics to reveal that the harsh environment of the inflammatory lesion drives neutrophils toward de novo protein synthesis and extracellular protein scavenging as a primary fuel. This study provides compelling evidence that tissue neutrophils scavenge extracellular proteins to fuel carbon metabolism, which aids in de novo protein synthesis and the promotion of an inflammatory phenotype. These observations reveal the surprisingly creative extent to which cells and tissues might adapt to energy-deficient inflammatory environments.
\end{abstract}

\section{Neutrophils are energetically active}

The acute inflammatory response is characterized by the rapid influx of large numbers of neutrophils into the tissue. Traditionally, it was believed that neutrophils only had three main roles: phagocytosis, degranulation, and release of nuclear material in the form of neutrophil extracellular traps (1). We now know that neutrophils are not restricted to these roles. Current research shows that they are a dynamic and adaptive cell type. Not only do neutrophils respond to signals, they also produce cytokines and inflammatory factors (2). Furthermore, they can actively mold the tissue microenvironment by rapidly depleting oxygen, secreting adenine nucleotides, and acidifying the extracellular space (3-5). Neutrophils are largely considered to be glycolytic, relying on glucose for ATP production (6). In this issue of the JCI, Watts et al. (7) showed that inflamed airways have substantially decreased extracellular glucose compared with healthy lungs, a finding also described in 2012 $(8,9)$. This observation raised the question that Watts and colleagues extensively explored: What do these energetically active immune cells use for a fuel source at sites of inflammation?

\section{Neutrophils under oxygen and glucose-deplete conditions}

Watts and colleagues used high-resolution mass spectrometry and showed that the most abundant proteins isolated from inflammatory neutrophils are associated with metabolic, migratory, inflammatory, and biosynthetic pathways (7). Given the observation that oxygen availability plays a key role in the innate immune response in lung injury and infection, the authors

\section{Related Article: https://doi.org/10.1172/JCl134073}

Conflict of interest: The authors have declared that no conflict of interest exists.

examined differences in the proteomes between neutrophils isolated from mice exposed to normoxia or hypoxia following lung injury. Hypoxia resulted in substantial increases in inflammatory receptors, including formylated peptide receptors, TNFRSF1b, and CSF2RB, in neutrophils. The observation that neutrophils isolated from hypoxic mice showed no reduction in intracellular levels of key granule proteins, despite detection of MMP9 and elastase, which are markers of degranulation, is of particular interest. The authors used a combination of heavy labelled amino acids and mass spectrometry to show de novo synthesis of granular proteins in neutrophils. Additionally, it was shown that neutrophils can uptake extracellular proteins and that heavy carbon from these extracellular proteins is incorporated into TCA cycle metabolites. Extensions of these studies revealed that neutrophils uptake proteins from the extracellular space through macropinocytosis, and that hypoxia enhanced protein uptake and proteolysis in inflammatory neutrophils (7).

To gain insight into the regulation of protein scavenging and breakdown, Watts et al. examined mammalian target of rapamycin (mTOR) and its associated complexes. In vitro, unstimulated neutrophils had low levels of mTORC activity and LPS stimulation resulted in a marked increase in mTORC activity. This observation was replicated in vivo and extended to show that both hypoxia and glucose deprivation inhibited mTORC activity. Neutrophils treated with a mTORC inhibitor had notable increases in proteolysis, suggesting neutrophils under oxygen- and glucose-deplete conditions would have enhanced proteolysis and an accentuated inflammatory phenotype (Figure 1). Having demonstrated the involvement of lysosomal trafficking in extracellular protein catabolism in vitro, the authors looked to confirm the physiological relevance of lysosomal 


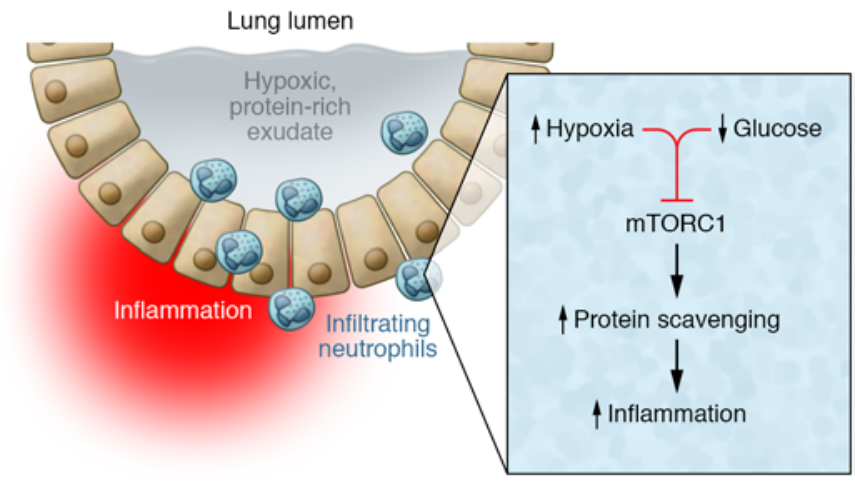

Figure 1. Lung neutrophils use protein as a primary energy source during active inflammation. During acute inflammation of the lung, the combination of increased hypoxia and decreased glucose availability inhibit mTORC1 in infiltrating neutrophils. As a result, neutrophils use available protein sources for energy production that drives a proinflammatory phenotype to enhance the inflammatory response.

protein trafficking in inflammation outcomes. Administration of chloroquine, an inhibitor of lysosomal acidification, reduced protein breakdown in neutrophils and suppressed neutrophil granule protease production following LPS stimulation in mice exposed to hypoxia (7).

\section{Conclusions and clinical implications}

Given the substantial presence of neutrophils at sites of inflammation and bystander tissue damage as a result of neutrophil degranulation, it is crucial that we gain a better understanding of the factors that influence neutrophil phenotypes. Within the lung, hypoxia appears to promote a damaging inflammatory neutrophil phenotype (9). On the basis of the work by
Watts et al., inhibition of neutrophil protein scavenging results in a less inflammatory tissue neutrophil and also reduces tissue damage in hypoxic lung injury models (7).

Importantly, hypoxia may not be detrimental in all tissue types. Within the colon, there exists a steep oxygen gradient across the colonic mucosa with epithelial cells in a state of physiological hypoxia $(10,11)$. Neutrophil migration into the colon promotes an increase in localized hypoxia and stabilization of hypoxia-inducible factor (HIF) to promote the resolution of inflammation $(12,13)$. These findings support the concept of using pharmacological agents to stabilize HIF (e.g., prolyl hydroxylase inhibitors) as a means of promoting wound healing and resolving inflammation in disorders such as inflammatory bowel disease (IBD). Preclinical models of IBD have shown promise in this regard (14). The lung and colon are, of course, vastly different organs. For example, they support quite different microbiomes and the availability of extracellular nutrients is quite different $(15,16)$. In future studies, it would be interesting to define what factors within the microenvironment influence the development of a proinflammatory neutrophil phenotype(s) and how protein utilization by neutrophils impacts inflammatory phenotypes in mucosal organs as well as organs that develop sterile inflammation (e.g., joints).

Having insight into the energetics and metabolism of hypoxic neutrophils could provide new opportunities to dampen acute inflammation. For example, Watts et al. demonstrate that glutamine from scavenged proteins provide an important energy source for hypoxic and sugar-starved neutrophils. It may be possible, therefore, to consider targeting the glutaminolysis pathway as a mechanism to inhibit the development of this proinflammatory neutrophils. Glutaminase was the most substantially induced enzyme in hypoxic neutrophils. Interestingly, several cancer clinical trials are underway with the glutaminase inhibitor CB-839. Targeting the glutaminolysis pathway is an area of active development (17) and based on Watts et al. (7), it would be interesting to consider whether glutaminase inhibitors may reduce inflammation in some cases.

\section{Acknowledgments}

This work was supported by grants from the National Institutes of Health (DK1047893, DK050189, DK095491) and by Veterans Administration Merit Award BX002182.

Address correspondence to: Sean P. Colgan, University of Colorado School of Medicine, 12700 East 19th Avenue, MS B-146, Aurora, Colorado 80045, USA. Phone: 303.724.7235; Email: sean.colgan@ cuanschutz.edu.
1. Rosales C. Neutrophil: a cell with many roles in inflammation or several cell types? Front Physiol. 2018;9:113.

2. Nauseef WM, Borregaard N. Neutrophils at work. Nat Immunol. 2014;15(7):602-611.

3. Campbell EL, et al. Transmigrating neutrophils shape the mucosal microenvironment through localized oxygen depletion to influence resolution of inflammation. Immunity. 2014;40(1):66-77.

4. Cartwright IM, et al. Adaptation to inflammatory acidity through neutrophil-derived adenosine regulation of SLC26A3. Mucosal Immunol. 2020;13(2):230-244.

5. Curtis VF, et al. Neutrophils as sources of dinucleotide polyphosphates and metabolism by epithelial ENPP1 to influence barrier function via adenosine signaling. Mol Biol Cell. 2018;29(22):2687-2699.

6. Borregaard N, Herlin T. Energy metabolism of human neutrophils during phagocytosis. J Clin Invest. 1982;70(3):550-557.

7. Watts ER, et al. Protein scavenging drives neutrophilic inflammation in hypoxia. J Clin Invest. 2021;131(10):e134073.

8. Garnett JP, et al. Proinflammatory mediators disrupt glucose homeostasis in airway surface liquid. J Immunol. 2012;189(1):373-380.

9. Sadiku P, et al. Prolyl hydroxylase 2 inactivation enhances glycogen storage and promotes excessive neutrophilic responses. J Clin Invest. 2017;127(9):3407-3420.

10. He G, et al. Noninvasive measurement of anatomic structure and intraluminal oxygenation in the gastrointestinal tract of living mice with spatial and spectral EPR imaging. Proc Natl Acad Sci US A. 1999;96(8):4586-4591.

11. Colgan SP, Taylor CT. Hypoxia: an alarm signal during intestinal inflammation. Nat Rev Gastro- enterol Hepatol. 2010;7(5):281-287.

12. Glover LE, et al. Oxygen metabolism and barrier regulation in the intestinal mucosa. JClin Invest. 2016;126(10):3680-3688.

13. Taylor CT, Colgan SP. Regulation of immunity and inflammation by hypoxia in immunological niches. Nat Rev Immunol. 2017;17(12):774-785.

14. Colgan SP, et al. Hypoxia and innate immunity: keeping up with the HIFsters. Annu Rev Immunol. 2020;38(doi):341-363.

15. Huffnagle GB, et al. The respiratory tract microbiome and lung inflammation: a two-way street. Mucosal Immunol. 2017;10(2):299-306.

16. Rooks MG, Garrett WS. Gut microbiota, metabolites and host immunity. Nat Rev Immunol. 2016;16(6):341-352.

17. Song M, et al. Recent development of small molecule glutaminase inhibitors. Curr Top Med Chem. 2018;18(6):432-443. 